\title{
MRTI-Based Optimization and Real-Time Laser Surgical Control for Cancer Treatment Using Fast Inverse Analysis Techniques
}

\author{
Yusheng Feng \\ Computational Bioengineering and Nanotechnology Lab \\ Department of Mechanical Engineering \\ The University of Texas at San Antonio \\ San Antonio, Texas, U.S.A. \\ yusheng.feng@utsa.edu \\ David Fuentes and Andrea Hawkins, and J. Tinsley Oden \\ Institute for Computational Engineering and Sciences \\ The University of Texas at Austin \\ Austin, Texas, U.S.A. \\ \{fuentes,ahawkins,oden\}@ices.utexas.edu
}

\begin{abstract}
For cancerous tumors in vital internal organs, minimally invasive laser surgery may be a desirable choice for cancer treatment due to its precise control and compatibility with most of the imaging modalities such as MRI (magnetic resonance imaging). However, the complexity of tumor composition and tissue response to a thermal dose demands real time optimization and control. In the previous work, we have developed a quite general computational framework that is capable of processing MRI anatomical data, providing pretreatment surgical protocol, and controlling tissue damage based on in vivo MRTI (magnetic resonance thermal imaging) data. In this paper, we describe computational techniques that are involved in real time optimization and control for laser surgical protocols of cancer treatment.
\end{abstract}

\section{Introduction}

Thermal therapies delivered by laser or other treatment modalities can provide minimally invasive cancer treatments that have the potential to become an effective option to eradicate the disease, maintain functionality of infected organs, and minimize complications and relapse. However, the ability to control the energy deposition to prevent damage to adjacent healthy tissue is a limiting factor in all forms of thermal therapies [9], including cryotherapy, microwave, radio-frequency, ultrasound, as well as laser. The combination of image guidance with computational prediction has the potential to allow unprecedented control over the bioheat transfer. Image guidance facilitates real time treatment monitoring through temperature feedback during treatment delivery $[8,10]$. Moreover, high performance numerical implementations of mathematical bioheat transfer models can be used to process in vivo thermal imaging data to assist treatment planning as well as to predict the outcome of the treatment for real-time surgical control [6]. This system is able to provide surgeons in a operating room with real-time feedback and prediction of optimal temperature and damage fields which will permit patient-specific optimized therapy (see figure 1).

\section{Biological background and Mathematical Models}

Laser-tissue interactions are characterized by optical response and the resulting thermal response. To simulate laser surgery and make reliable predictions of the temperature field requires two major modeling components: a bioheat transfer model for the tissue and a laser source term that characterizes thermal energy deposited into the tissue. Thermal damage processes in cells and tissues are usually quantified by kinetic models based on a first-order rate process to characterize pathological transformation to specific states by observable alterations such as coagulation or desiccation. The mathematical representation of the temperature distribution in the tissue is usually formulated with Pennes bioheat equation for the thermal effects of local blood perfusion. The laser as a heating source can be model 


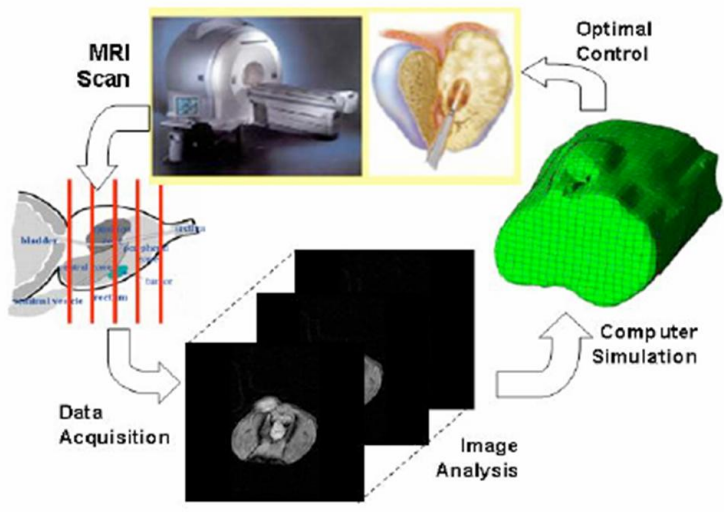

Figure 1. Illustration of a MRTI-Guided Feedback Control System

either by an isotropic term or Monte Carlo method. Both cell damage, as a major indicator of treatment outcome, is characterized by the traditional Arrhenius law for a given temperature filed. The laser source can be applied near the treatment surface or placed interstitially inside a tumor.

Let $\Omega$ be a bounded domain (tumor region) in $\mathcal{R}^{3}$ with $\Gamma=\partial \Omega_{\mathrm{C}} \bigcup \partial \Omega_{\mathrm{N}}$ denoting a Lipschitz continuous boundary. Eqn. (1) is a generalized Pennes bioheat equation with nonlinear coefficients.

$\rho c_{p} \frac{\partial T}{\partial t}-\nabla \cdot(k(T) \nabla T)+\omega(T) c_{b}\left(T-T_{a}\right)=Q(\mathbf{x}, t) \quad$ in $\Omega$

The thermal conductivity, $k\left[J s^{-1} m^{-1} K^{-1}\right]$, and blood perfusivity, $\omega\left[\mathrm{kg} \mathrm{s}^{-1} \mathrm{~m}^{-3}\right]$, are assumed to be bounded functions of the temperature field, $T=T(\mathbf{x}, t)$. The specific heat of the tissue and blood are given by $c_{p}$ and $c_{b}$ respectively, $T_{a}$ is the arterial temperature, and $\rho$ is the density of the tissue. On the Cauchy boundary,

$$
-k(T) \nabla T \cdot \mathbf{n}=h\left(T-T_{\infty}\right) \quad \text { on } \partial \Omega_{\mathrm{C}}
$$

The coefficient of cooling is denoted $h . T_{\infty}$ denotes the ambient temperature. $\mathcal{G}$ is the prescribed heat flux on the Neumann boundary.

$$
-k(T) \nabla T \cdot \mathbf{n}=\mathcal{G} \quad \text { on } \partial \Omega_{\mathrm{N}}
$$

The temperature field is propagated forward in time from a given initial condition, $T_{\mathrm{o}}$.

$$
T(\mathbf{x}, 0)=T_{\circ} \quad \text { in } \Omega
$$

The laser-tissue interaction is a complex phenomenon that involves both optical and thermal responses [?, ?]. Since laser light is absorbed by tissue and converted into heat, a commonly used model that characterize the absorbed heat can be represented by the rate of heat generation $Q(\mathbf{x}, t)$, which is defined as

$$
\begin{aligned}
Q(\mathbf{x}, t) & =\mu_{a} \Phi(\mathbf{x}, t) \\
& =\mu_{a} \mu_{t r} P(t) \frac{3 \exp \left(-\mu_{e f f}\left\|\mathbf{x}-\mathbf{x}_{\circ}\right\|\right)}{4 \pi\left\|\mathbf{x}-\mathbf{x}_{\circ}\right\|}
\end{aligned}
$$

where $\mu_{t r}=\mu_{a}+\mu_{s}(1-g), \mu_{e f f}=\sqrt{3 \mu_{a} \mu_{t r}}$, and $\Phi(\mathbf{x}, t)$ is the fluence that defines the amount of energy, in the form of photons, passing through a unit area at a point in space per unit time. $P(t)$ is the laser power at time $t$. The parameters $\mu_{a}, \mu_{s}$ are absorption and scattering coefficients that relate to laser wavelength. The constant $g$ is the anisotropic factor, and $\mathbf{x}_{\circ}$ is the position of the laser source.

However, Eqn. (2) is valid when the scattering coefficient is much larger than the absorption coefficient $\left(\mu_{s} \ll \mu_{a}\right)$ and when the laser source is far away from the boundary. Moreover, this model only accounts for scattered light, i.e., the primary light is ignored. To better characterized lasertissue interaction, we also employ a Monte Carlo method to compute the fluence. There two major assumptions made in order to construct the Monte Carlo model: (a) the photons will be distributed in a cylindrically symmetric manner with the initial direction of the beam as the axial direction, and (b) the probability distributions being used for the scattering angles and mean free path length are known. Generally speaking, Monte Carlo method is considered to be more accurate but computationally expansive than the classical approach.

\section{Brief Description of the Computational Framework}

\subsection{Pre-treatment Planning}

Prior to laser treatment of a tumor region, the location of optical fiber and laser power are optimized to control the temperature in the tissues such that cancer cells in the cancerous tissue region are destroyed or sensitized and surrounding tissue damage is minimized. During treatment, intra-operative MRI data is used to register the computational domain with the biological domain and real-time thermal imaging MRTI data drives the calibrations aligning the parameters of the bioheat transfer model to the patient's biological tissue values. As new thermal imaging data is acquired intermittently (every 5 seconds), the computational prediction is compared to the measurements of the real-time thermal images and the observed differences in the temperature field are used to update the computations to drive laser parameter optimization as well as computational mesh adaption [7]. 
The software infrastructure is built from the Petsc [1] parallel computing paradigm and the Toolkit for Advanced Optimization (TAO) [2] parallel optimization library. Advanced Visual Systems (AVS) [5] is used in conjunction with a Virtual Network Computing (VNC) server for remote visualization. AVS coroutines are used to manage and coordinate the simultaneous visualization of the MRI anatomical image, MRTI thermal image, and finite element data sets. The Imaging-to-Modeling software system for anatomical MRI data employs both image processing and geometry processing functionalities to produce a suitable linear or higher order meshed model of the anatomy.

\subsection{Real Time Imaging Acquisition and Process}

The magnetic resonance temperature imaging (MRTI) data are acquired every five seconds. However, the raw imaging data is often of poor quality which makes it difficult to build a quality meshed model of the anatomy under investigation. In order to improve the image quality, we have developed a suite of image processing functionalities that facilitate further processing. The modules encapsulated in the image processing units are: (1) contrast enhancement (2) filtering (3) segmentation (4) co-registration, and (5) mesh generation.

\section{Fast Inverse Analysis and Optimal Control}

The goal of the temperature-based optimal control laser treatment of cancer by oblation and its associated calibration problems within the control loop is to find the set of model parameters that minimize the space-time norm of the difference between the computed temperature field $T(\beta, \mathbf{x}, t)$ and an ideal field $T_{\text {ideal }}(\mathbf{x}, t)$. The mathematical formulation can be formally stated as follows:

Find the model coefficients, $\beta^{*} \in \mathbb{P}$, that produces the temperature field, $T^{*} \in \mathcal{V}$, such that

$$
\begin{aligned}
Q\left(T^{*}\left(\beta^{*}\right), \beta^{*}\right) & =\frac{1}{2} \int_{0}^{\tau} \int_{\Omega} \chi(\mathbf{x})\left(T^{*}(\beta, \mathbf{x}, t)\right. \\
& \left.-T_{\text {ideal }}(\mathbf{x}, t)\right)^{2} d x d t+\Phi(\beta)
\end{aligned}
$$

satisfies

$$
\begin{aligned}
& Q\left(T^{*}\left(\beta^{*}\right), \beta^{*}\right)=\inf _{\beta \in \mathbb{P}} Q(T(\beta), \beta) \\
& \quad \mathbb{P}=\{\beta: \exists ! T \text { s.t. } C(u, \beta ; v)=0 \quad \forall v \in \mathcal{V}\}
\end{aligned}
$$

The ideal field for the calibration problem is the experimentally determined temperature field $T_{\exp }(\mathbf{x}, t)$. The ideal field for temperature based optimal control is given as follows.

$$
u_{\text {ideal }}= \begin{cases}37.0^{\circ} \mathrm{C} & \mathbf{x} \in \Omega_{H} \\ 54.0^{\circ} \mathrm{C} & \mathbf{x} \in \Omega_{C}\end{cases}
$$

The function $\Phi(\beta)$ is a penalty term added to the objective function used to keep the model parameters within physically acceptable bounds.

$$
\begin{aligned}
\Phi(\beta) & =\sum_{i=1}^{N_{\text {params }}} \exp \left(\gamma \frac{\beta_{i}-\beta_{i}^{U B}}{\beta_{i}^{U B}-\beta_{i}^{L B}}\right) \\
& +\exp \left(-\gamma \frac{\beta_{i}-\beta_{i}^{L B}}{\beta_{i}^{U B}-\beta_{i}^{L B}}\right)
\end{aligned}
$$

where $\beta_{i}$ denotes a vector of particular parameter set. The dimension of the parameter space is denoted $N_{\text {params }}$. The physically acceptable lower and upper bounds of the parameter, $\beta_{i}$, is denoted by $\beta_{i}^{L B}$ and $\beta_{i}^{U B}$, respectively. The penalty term is scaled by $\gamma=1000.0$.

The optimization problem in (3) is solved using an adjoint method to compute the gradient of the quantity of interest. The following Galerkin representation of the temperature field and adjoint variable is assumed.

$$
\begin{aligned}
T(\mathbf{x}, t) & =\sum_{k=1}^{N_{\text {step }}} \sum_{j=1}^{N_{\text {dof }}} \alpha_{j}^{k}(t) \phi_{j}(\mathbf{x}) \\
p(\mathbf{x}, t) & =\sum_{k=1}^{N_{\text {step }}} \sum_{i=1}^{N_{\text {dof }}} \lambda_{i}^{k}(t) \phi_{i}(\mathbf{x})
\end{aligned}
$$

where $N_{\text {step }}$ is the number of time steps, $N_{\text {dof }}$ is the number of Galerkin coefficients, and $\phi_{i}$ 's are the finite element basis functions of polynomial order $\mathrm{p}(=1,2,3 \ldots)$. Functions $\alpha_{j}^{k}(t)$ and $\lambda_{i}^{k}(t)$ are coefficients associated with primary variable $T(\mathbf{x}, t)$ and adjoint variable $p(\mathbf{x}, t)$, respectively. The time discretization of the laser power is assumed in a form of piecewise constant in time.

$$
P(t)=\left\{\begin{aligned}
P_{k}, & t \in\left[t_{k-1}, t_{k}\right) \\
0, & \text { otherwise }
\end{aligned}\right.
$$

Solving for the adjoint variable, $p_{k}$ at $t=t_{k}$ time step, for inverse analysis and optimal control requires the numerical gradient of the quantity of interest to be computed as follows.

$$
\frac{\partial Q(T, \beta)}{\partial \beta}=\sum_{k=1}^{N_{\text {step }}}\left(\begin{array}{c}
-\Delta t_{k} \int_{\Omega} \frac{\partial k}{\partial \beta}\left(T_{k-\frac{1}{2}}, \mathbf{x}, \beta\right) \nabla T_{k-\frac{1}{2}} \\
\cdot \nabla p_{k} d x \\
-\Delta t_{k} \int_{\Omega} c_{\text {blood }} \frac{\partial \omega}{\partial \beta}\left(T_{k-\frac{1}{2}}, \mathbf{x}, \beta\right) \\
\left(T_{k-\frac{1}{2}}-T_{a}\right) p_{k} d x \\
+\Delta t_{k} \int_{\Omega} \frac{\partial Q_{\text {laser }}}{\partial \beta}\left(\beta, \mathbf{x}, t_{k}\right) p_{k} d x
\end{array}\right)
$$


We use the same computational structure to compute both primary and adjoint problems. The implementation involves the nonlinear transient bioheat transfer model using a finite element discretization in space and finite difference in time using the Crank-Nicolson scheme. In order to meet the goal of real time control, we employ efficient parallel computing algorithms to achieve the goal that each run only takes a few seconds using Linux clusters operated by Texas Advanced Computing Center (TACC) in Austin, Texas.

\section{MRTI Measurement and Computational Results}

Data acquired for calibration are obtained from MRI experiments conducted at M.D. Anderson Cancer Center in Houston, which consist MRTI images of a mouse tumor with resolution of $49 \times 56$ pixel. The field of view is $4 \times 6$ $\mathrm{cm}^{2}$ and the thickness associated with the image is $3 \mathrm{~mm}$. An external heat source was applied to a mouse tumor. Sixty thermal images were acquired at every 5 seconds.

The computational results presented in this section is similar to the thermal imaging data studied in [4] and [6]. The calibration algorithm is applied to these data sets to invert for the constitutive equation nonlinearites as well as the heterogeneity of the biological domain.

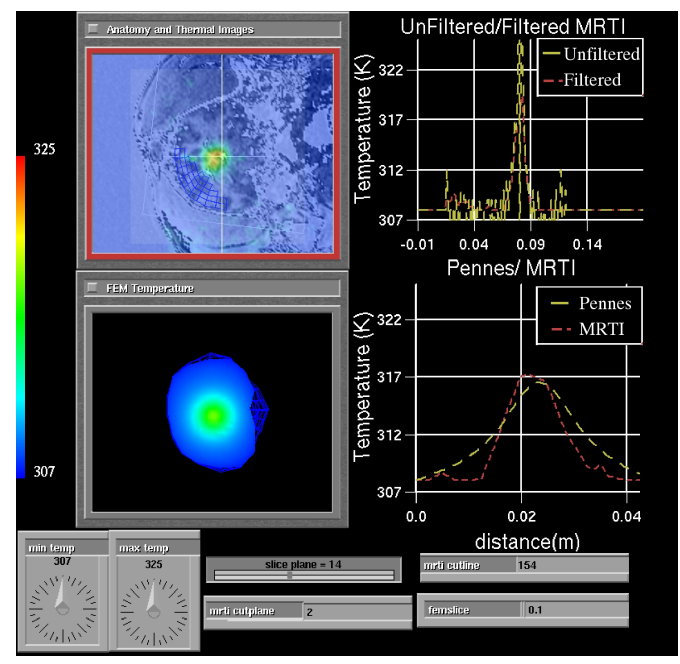

Figure 2. Images of MRTI in vivo Measurement and FEM Computation of a Canine Brain

The effect of calibrating the nonlinear perfusion, $\omega(T, \mathbf{x}, \beta)$, and thermal conductivity, $k(T, \mathbf{x}, \beta)$, parameters in Pennes model was studied. Calibration was done with respect to MRTI thermal imaging data of in vivo heating of canine brain tissue. The thermal imaging data was acquired in the form of five two-dimensional $256 \times 256$ pixel

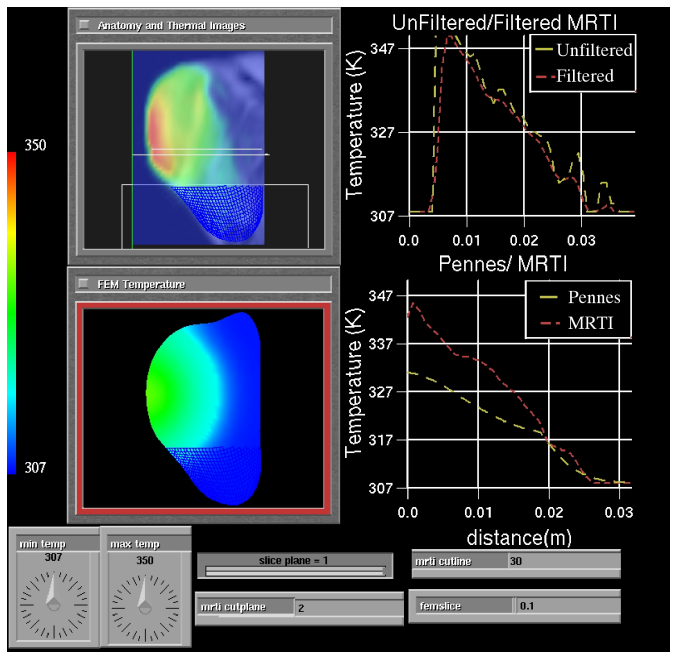

Figure 3. Images of MRTI in vivo Measurement and FEM Computation of a Tumor Grown on the Hind Leg of a Mouse

images every six seconds for 120 time steps. The spacing between images was $3.5 \mathrm{~mm}$. A manual craniotomy of a canine skull was preformed to allow insertion of an interstitial laser fiber to provide the heating. A template based finite element mesh was generated from thirty-six; two-dimensional $256 \times 256$ pixels MRI images of the canine brain. The field view was $200 \times 200 \mathrm{~mm}^{2}$ with each image spaced at $1 \mathrm{~mm}$ apart. The FEM prediction using linear material coefficients [3], $k(T, \mathbf{x}, \beta)=0.527\left[\frac{\mathrm{W}}{\mathrm{mK}}\right]$ and $\omega(T, \mathbf{x}, \beta)=6.0\left[\frac{\mathrm{kg}}{\mathrm{sm}^{3}}\right]$, is shown in Figure 2. The upperright windows in Figures 2 shows a cut-line comparison of the filtered MRTI data with the unfiltered data. The upperleft windows in Figure 2 display an overlay of the MRTI thermal image onto the anatomical MRI image. At the lower-left window, it shows a 2D temperature slice through the 3D domain. Figure 2 was obtained by inverting for the constitutive vectors. As shown in [6], Figures 2 illustrates that the material nonlinearities are necessary to model the late time heat dissipation. The results indicate that a spatially varying inversion for the perfusion field should provide a means to further increase the accuracy of the FEM temperature prediction of the canine brain data.

Allowing the perfusion and thermal conductivity model parameters to vary as a spatial field is seen to have a tremendous effect on the model calibrations. Inverting for the spatial variation in the parameters embeds the biological tissue heterogeneity within the Pennes model. Imaging data of an external laser applied to a tumor grown on a mouse's hind leg was used to study the effect of the parameter field inversion. Sixty thermal images were acquired at an interval of five seconds. A single time instance of the data is shown in 
Figures 3. The field of view is $4 \times 6 \mathrm{~cm}^{2}$ and the thickness associated with the MRI/MRTI images is $3 \mathrm{~mm}$. Figures 3 compare the FEM prediction using text book linear material coefficients to the calibrated heterogeneous material coefficients applied to the in vivo heating of a tumor grown on a mouse. The upper-right windows in Figures 3 each show a cut-line comparison of the filtered MRTI data with the unfiltered data. The upper-left windows in Figures 3 display an overlay of the MRTI thermal image onto the anatomical MRI image. The images are $49 \times 56$ pixels. Figure 3 lowerleft window shows a $2 \mathrm{D}$ temperature slice through the $3 \mathrm{D}$ domain. The lower-right window is a cut-line comparison of the filtered MRTI data to the FEM prediction.

The agreement between the predicted FEM solution and the MRTI thermal images shown in Figure 3 illustrates the importance of inverting for the field of material heterogeneity. Figure 3 represents a 4100-parameter optimization problem, which is very computational intensive. The initial guess for the material coefficients was assumed homogeneous. The optimization automatically determines the heterogeneity (spatial variation of the parameters) that allows Pennes model to accurately predict the temperature field as observed in the experiments. This result shows that the optimization through evolution of the material heterogeneity inversion for the thermal conductivity fields and blood perfusion fields is very effective.

The system has been tested on a $1 \%$ agar phantom material. This testing represents a project milestone. The phantom material has provided an animal-free method of testing and debugging the entire control system. For testing purpose, the thermal images were mainly used to periodically update the initial condition of the FEM computation in real time. After each update, the calculations proceeded to predict all the way to the end of the treatment. On current code implementation on parallel computing architectures, we have the capability to produce 40 to 50 seconds of predictions using only 10 seconds of computation time. It means that the current system satisfies the requirement for real-time control with 30 to 40 seconds leading time.

\section{Conclusions}

In this paper, we have presented a fast inverse analysis technique that was implemented in parallel to solve bio-heat transfer problems for cancer treatment so that the real-time control is made possible. Another important factor in the case of laser therapy involves modeling the laser fluence in the tissue correctly. While there are two standard ways of modeling this, analytically or by using a Monte Carlo method, they both depend on three optical parameters of interest: the absorption coefficient, $\mu_{a}$, the scattering coefficient, $\mu_{s}$, and the anisotropic factor, $g$. In living tissue, each of these parameters is truly a function of space, light wavelength, and temperature. The validation tests demonstrate an accurate and robust model can produce accurate results in satisfactory time for real-time control.

Acknowledgments. We wish to thank our DDDAS team members (http://dddas.ices.utexas.edu) for their collaborative efforts and contributions in MRI/MRTI data collection, cell damage characterization, and mesh generations. The research in this paper was supported in part by the National Science Foundation (CNS-0540033).

\section{References}

[1] S. Balay, W. D. Gropp, L. C. McInnes, and B. F. Smith. Petsc users manual. Technical Report ANL-95/11 - Revision 2.1.5, Argonne National Laboratory, 2003.

[2] S. J. Benson, L. C. McInnes, J. Moré, and J. Sarich. TAO user manual (revision 1.8). Technical Report ANL/MCS-TM-242, Mathematics and Computer Science Division, Argonne National Laboratory, 2005. http://www.mcs.anl.gov/tao.

[3] K. R. Diller, J. W. Valvano, and J. A. Pearce. Bioheat transfer. In F. Kreith and Y. Goswami, editors, The CRC Handbook of Mechanical Engineering, 2nd Ed., pages 4-278-4357. CRC Press, Boca Raton, 2005.

[4] Y. Feng, D. Fuentes, A. Hawkins, J. Bass, M. N. Rylander, A. Elliott, A. Shetty, R. J. Stafford, and J. T. Oden. Nanoshell-mediated laser surgery simulation for prostate cancer treatment. Special Issue on Computational Bioengineering, Engineering with Computers, Accepted for publication, 2007

[5] A. V. S. Inc. Avs user's guide, May 1992. National Congress on Computational Mechanics. San Francisco, California, Conference Presentation.

[6] J. T. Oden, K. R. Diller, C. Bajaj, J. C. Browne, J. Hazle, I. Babuška, J. Bass, L. Demkowicz, Y. Feng, D. Fuentes, S. Prudhomme, M. N. Rylander, R. J. Stafford, and Y. Zhang. Dynamic data-driven finite element models for laser treatment of prostate cancer. Num. Meth. PDE, 23 : 904-922, 2007.

[7] J. T. Oden and S. Prudhomme. Goal-oriented error estimation and adaptavity for the finite element method. Computers and Mathematics with Applications, 41(5-6):735-756, 2001.

[8] R. Salomir et al. Hyperthermia by MR-guided focused ultrasound: accurate temperature control based on fast MRI and a physical model of local energy deposition and heat conduction. Magn. Reson. Med., 43(3):342-347, 2000.

[9] K. Shinohara. Thermal ablation of prostate diseases: advantages and limitations. Int. J. Hyperthermia, 20(7):679-697, 2004.

[10] F. C. Vimeux et al. Real-time control of focused ultrasound heating based on rapid MR thermometry. Invest. Radiol., 34(3):190-193, 1999. 